\title{
The relative effects of corneal thickness and age on Goldmann applanation tonometry and dynamic contour tonometry
}

\author{
A Kotecha, E T White, J M Shewry, D F Garway-Heath
}

Br J Ophthalmol 2005;89:1572-1575. doi: 10.1136/bjo.2005.075580

Aims: To establish the effects of central corneal thickness (CCT) on intraocular pressure (IOP) measured with a prototype Pascal dynamic contour tonometer (DCT), to evaluate the effect of CCT and age on the agreement between IOP measured with the Pascal DCT and Goldmann applanation tonometer (GAT), and to compare the interobserver and intraobserver variation of the DCT with the GAT.

Methods: GAT and DCT IOP measurements were made on 130 eyes of 130 patients and agreement was assessed by means of Bland-Altman plots. The effect of CCT and age on GAT/DCT IOP differences was assessed by linear regression analysis. Interobserver and intraobserver variations for GAT and DCT were assessed in 100 eyes of 100 patients.

Results: The mean difference (95\% limits of agreement) between GAT and DCT was $-0.7(-6.3$ to 4.9$) \mathrm{mm} \mathrm{Hg}$. GAT/DCT IOP differences increased with thicker CCT (slope $0.017 \mathrm{~mm} \mathrm{Hg} / \mu \mathrm{m}, 95 \% \mathrm{Cl} 0.004$ to $0.03, r^{2}=0.05$, $p=0.01$ ), and with greater age, slope $0.05 \mathrm{~mm} \mathrm{Hg} /$ year (95\% Cl 0.012 to $0.084, r^{2}=0.05, p=0.01$ ). The intraobserver variability of GAT and DCT was $1.7 \mathrm{~mm} \mathrm{Hg}$ and $3.2 \mathrm{~mm} \mathrm{Hg}$, respectively. The interobserver variability was (mean difference (95\% limits of agreement)) $0.4(-3.5$ to 4.2) $\mathrm{mm} \mathrm{Hg}$ for GAT and 0.2 ( -4.9 to 5.3 ) $\mathrm{mm} \mathrm{Hg}$ for DCT. Conclusions: GAT is significantly more affected than DCT by both CCT and subject age. The effect of age suggests an age related corneal biomechanical change that may induce measurement error additional to that of CCT. The prototype DCT has greater measurement variability than the GAT.

$\mathrm{T}$ he current "gold standard" for intraocular pressure (IOP) measurement is the Goldmann applanation tonometer (GAT), which makes a static measurement of the force required to flatten a fixed area of the cornea. In designing the tonometer, Goldmann recognised that corneal effects, such as central corneal thickness (CCT) and the precorneal tear film, would influence the pressure measurements, and this has been shown in various studies. ${ }^{1-6}$

A new slit lamp mounted tonometer, the Pascal dynamic contour tonometer (DCT; Swiss Microtechnology AG, Port, Switzerland) has been developed to remove the corneal biomechanical effects from IOP measurement by using a direct transcorneal method. When the Pascal DCT is placed on the eye, the cornea takes the contour of the tip so that its biomechanical effects on IOP measurement are reduced. The DCT gathers 100 IOP readings per second. Recent studies have indicated that its IOP measurements are independent of $\mathrm{CCT}^{7}$ and are unchanged following thinning of the CCT with laser in situ keratomileusis (LASIK).$^{8}$
The primary purpose of this study was to assess the agreement between the GAT and Pascal DCT, and to establish the effects of CCT on IOP measurements made with these two devices. A secondary aim was to evaluate the intraobserver and interobserver variability of the Pascal DCT.

\section{METHODS}

Patients attending their routine appointment in the ocular hypertension or pigment dispersion clinics at the Glaucoma Research Unit (Moorfields Eye Hospital, London, UK) between February and May 2004 were invited to take part in the study (see table 1 for demographic data). Informed consent, according to the tenets of the Declaration of Helsinki, was obtained before examination. The study had local ethics committee approval.

For the tonometer comparison study, 130 eyes of 130 patients were examined; 71 patients $(55 \%)$ were on topical ocular hypotensive therapy (table 2). Two GAT and three DCT (prototype version 1.2) readings were obtained in a randomised order. Measurements were performed by either one of two technicians (ETW or JMS) and by a clinician (AK) also in a randomised order. With GAT measurements, the tonometer was set at $10 \mathrm{~mm} \mathrm{Hg}$ before each reading. For DCT, three readings of "good" quality were saved (score $\leqslant 3$ on a scale up to 5, as recommended by the manufacturer). The observers were masked to each other's results. Keratometry was performed with the IOLMaster (Carl Zeiss Meditec, AG, Germany) before tonometry, and CCT was measured with the Altair ultrasonic pachymeter $(20 \mathrm{MHz}$ solid tip probe; Optikron 2000, Rome, Italy) after tonometry. The sample size chosen allows a study power of $90 \%(\mathrm{p}<0.05)$ to detect a correlation of $\mathrm{r}=0.3$ between CCT and IOP.

For the intraobserver and interobserver variability study, 100 eyes (49 left eyes) of 100 (45 female) patients were examined. The mean patient age was 60 years (range 26-83; SD 13.2 years). This group was a subset of that used in the previous study. The order of GAT/DCT and technician/ clinician was randomised with a 5 minute break between GAT and DCT measurements. Measurements were obtained as already described, and the agreement between technicians and clinician was assessed.

\section{Data analysis}

Bland-Altman plots ${ }^{10}$ were used to assess the agreement in IOP measurements between techniques (DCT measurements by the clinician versus GAT measurements by the technicians), and between observers for each of the two techniques. Mean difference and 95\% limits of agreement were calculated. Linear regression analysis was used to

Abbreviations: CCT, central corneal thickness; DCT, dynamic contour tonometer; GAT, Goldmann applanation tonometer; IOP, intraocular pressure; LASIK, laser in situ keratomileusis 


\begin{tabular}{llll}
$\begin{array}{l}\text { Table } 1 \\
(\mathrm{n}=130)\end{array}$ & Demographic data of comparison group \\
\hline & Mean & SD & Range \\
\hline Eye (left/number) & 60 & & \\
Sex (female/number) & 52 & & \\
Age (years) & 61 & 13.3 & $22-83$ \\
CCT ( $\mu \mathrm{m})$ & 556 & 36.9 & $468-642$ \\
Keratometry (mm) & 7.74 & 0.21 & $7.10-8.44$ \\
Corneal astigmatism (mm) & 0.17 & 0.13 & $0-0.88$ \\
GAT (mm Hg) & 19 & 4.6 & $9-33$ \\
DCT (mm Hg) & 19 & 4.0 & $11-29$ \\
\hline
\end{tabular}

determine the associations between GAT/DCT differences, and CCT and age.

Repeatability (intraobserver variability) of IOP measurements with GAT and DCT was calculated as 2.77 times the within subject standard deviation (wSSD $)^{11}$ :

$$
w s S D=S D(\text { observation } 1-\text { observation } 2) \sqrt{2}
$$

where $S D$ is the standard deviation, and observations 1 and 2 are the recorded IOP measurements. The wSSD was only calculated if the magnitude of SD was unrelated to that of mean IOP readings.

To establish the effect of the choice of reading, repeatability was calculated for DCT readings 1 and 2, and 2 and 3. The repeatability of GAT and DCT measurements was calculated for the clinician and technicians.

To assess the effect of DCT reading quality on repeatability, the association between the SD of DCT IOP measurements and average reading quality was determined.

All statistical analyses were performed using Medcalc Version 7.4.2.0 (Medcalc Software, Mariakerke, Belgium).

\section{RESULTS}

IOP measurements satisfying the quality criteria were obtained for all patients. Tables 1 and 2 summarise the demographic data of the study group.

\section{Agreement between GAT and DCT measurements}

The average of two GAT readings was compared with that of DCT readings 2 and 3 (see "Intraobserver and interobserver variability," below). The mean difference (95\% limits of agreement) between GAT and DCT was -0.7 ( -6.3 to 4.9 ) $\mathrm{mm} \mathrm{Hg}$, and no relation between GAT/DCT differences and average was found (fig 1).

\section{Effect of CCT on GAT and DCT IOP measurements}

There was a relation between GAT/DCT IOP differences and CCT (slope $0.017,95 \%$ CI 0.004 to $0.03, r^{2}=0.05, p=0.01$ ) (fig 2). Analysing treated and untreated eyes separately showed no relation between GAT/DCT IOP differences and

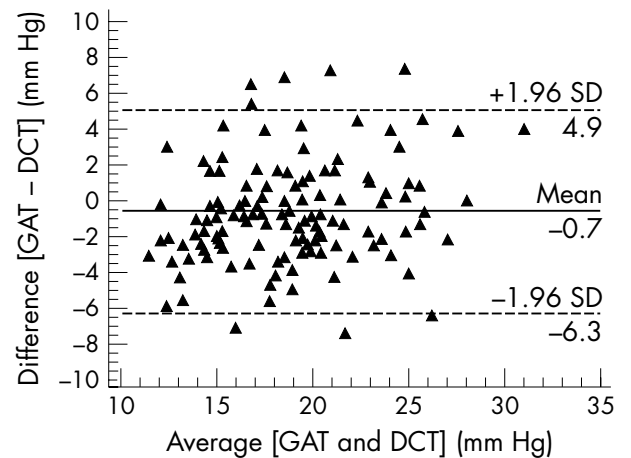

Figure 1 Bland-Altman plot of GAT/DCT IOP differences against GAT/ DCT mean.

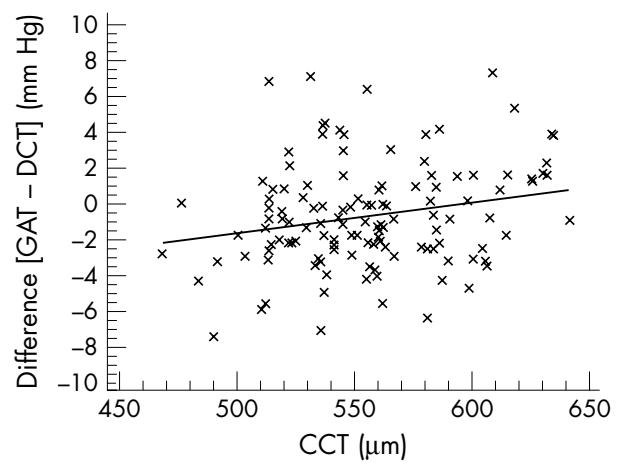

Figure 2 Relation between CCT and GAT/DCT IOP differences.

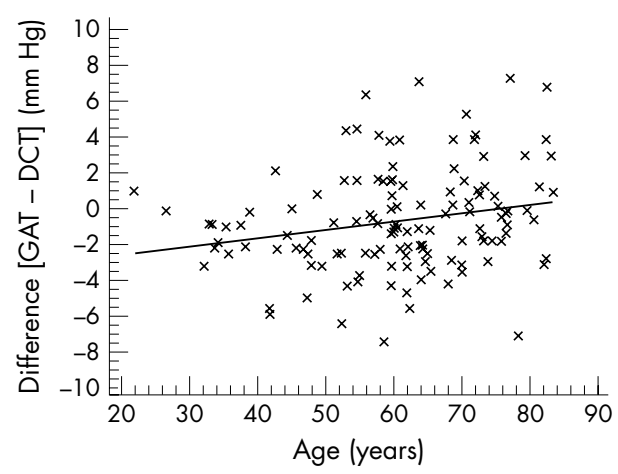

Figure 3 Relation between age and GAT/DCT IOP differences.

Table 2 Status and treatment of "comparison" group (total $n=130$, treated eyes $n=71$ )

\begin{tabular}{llllll}
\hline Diagnosis & $\begin{array}{l}\text { No of eyes } \\
\text { (number } \\
\text { treated) }\end{array}$ & $\begin{array}{l}\text { Prostaglandin } \\
\text { analogue } \\
\text { (number) }\end{array}$ & $\begin{array}{l}\boldsymbol{\beta} \text { blocker } \\
\text { (number) }\end{array}$ & $\begin{array}{l}\boldsymbol{\alpha} \text { agonist } \\
\text { (number) }\end{array}$ & $\begin{array}{l}\text { CAI } \\
\text { (number) }\end{array}$ \\
\hline OHT & $29 / 58$ & 15 & 12 & 0 & 2 \\
POAG & $21 / 21$ & 17 & 2 & 1 & 1 \\
PDS & $14 / 30$ & 7 & 6 & 1 & 0 \\
Glaucoma suspect & $7 / 21$ & 3 & 3 & 1 & 0 \\
\hline OHT, ocular hypertension; POAG, primary open angle glaucoma; PDS, pigment dispersion syndrome.
\end{tabular}


CCT in treated eyes $(\mathrm{n}=71$, CCT slope $0.004,95 \%$ CI 0.012 to $\left.0.020, r^{2}=0.003, p=0.65\right)$, but an association in untreated eyes $(\mathrm{n}=59$, CCT slope $0.025 ; 95 \%$ CI 0.003 to 0.046 , $r^{2}=0.083, \mathrm{p}=0.03$ ). Analysis of variance (ANOVA) performed to test whether treatment status had an effect on either CCT or GAT/DCT differences for the whole dataset showed no significant effect (F ratio $0.23, \mathrm{p}=0.6$ for CCT and F ratio 1.7, $\mathrm{p}=0.2$ for GAT/DCT difference).

\section{Effect of age on GAT and DCT IOP measurements}

GAT/DCT differences were associated with age (GAT/DCT difference $=0.05 \times$ age -3.6 , slope $95 \%$ CI 0.012 to 0.084 , $r^{2}=0.05, \mathrm{p}=0.01$ ) (fig 3). There was no relation between GAT/DCT IOP differences and age in treated eyes $(\mathrm{n}=71$, age slope $-0.031,95 \%$ CI -0.086 to $0.024, r^{2}=0.018 ; p=0.27$ ), whereas an association was found in untreated eyes $(\mathrm{n}=59$, age slope $0.079,95 \%$ CI 0.029 to $0.130, r^{2}=0.14, p=0.003$ ). Linear regression analysis showed no significant association between CCT and age (slope $0.174,95 \%$ CI 0.65 to -0.31 , $\left.r^{2}=0.004, p=0.48\right)$. Multiple regression analysis demonstrated an association between GAT/DCT differences, and both CCT and age (GAT/DCT difference $=0.045 \times$ age +0.016 $\times$ CCT $-12.5, r^{2}=0.09, p=0.01$ for both age and CCT).

\section{Intraobserver and interobserver variability}

With both techniques, IOP reading SDs were independent of the mean IOP. The repeatability of IOP measurements for each observer are summarised in table 3. The difference between two measurements on the same subject would be less than the value for repeatability in $95 \%$ of pairs of observations.

The repeatability of DCT measurements improved when the first reading was discarded. For this reason, the average of readings 2 and 3 was used for the interobserver variability study. The mean difference (95\% limits of agreement) in average GAT readings between clinician and technician was $0.4(-3.5$ to 4.2$) \mathrm{mm} \mathrm{Hg}$. The mean difference (95\% limits of agreement) in average DCT measurements between clinician and technician was $0.2(-4.9$ to 5.3$) \mathrm{mm} \mathrm{Hg}$. The association between DCT recording quality and DCT measured IOP variability approached, but did not reach, significance (Pearson $r=0.18 ; 95 \%$ CI -0.02 to $0.37 ; \mathrm{p}=0.08$ ).

\section{DISCUSSION}

In this study, a significant positive association between GAT/ DCT IOP differences and CCT was found. The association of CCT with GAT/DCT IOP differences was studied as 55\% of our subject population were on topical hypotensive therapy, which may confound the effect of CCT on IOP measurements. The association was shown not to be affected by treatment status, although a separate analysis indicated that GAT/DCT differences in untreated eyes were more affected by CCT compared with treated eyes. This finding, however, was not significant, perhaps because of the small subject numbers.

DCT IOP measurements have been reported to be independent of CCT, ${ }^{89}$ while studies have shown an increase in GAT IOP measurements with CCT, with slopes of $0.23 \mathrm{~mm} \mathrm{Hg}^{12}$ and $0.27 \mathrm{~mm} \mathrm{Hg}^{13}$ increase per $10 \mu \mathrm{m}$ increase

Table 3 Repeatability of measurement technique $(\mathrm{mm} \mathrm{Hg})$

\begin{tabular}{lll}
\hline Method & Technician & Clinician \\
\hline GAT 1 and 2 & 1.6 & 1.7 \\
DCT 1 and 2 & 4.2 & 3.3 \\
DCT 2 and 3 & 3.2 & 2.6 \\
\hline
\end{tabular}

in CCT reported in UK populations. Our findings indicate a slope of $0.17 \mathrm{~mm} \mathrm{Hg}$ per $10 \mu \mathrm{m}$ increase in CCT. A steeper slope would be expected if the DCT was not affected by CCT, and a study with greater power is required to test the hypothesis that CCT has a weak effect on DCT measured IOP. It is also possible that the association between IOP measurement and CCT is affected by a change in corneal biomechanics through the use of topical hypotensive therapy, especially prostaglandin analogues. Prostaglandin analogues are known to alter the extracellular matrix of the ciliary muscle, ${ }^{14}$ and they may also affect the extracellular matrix of the cornea, altering its rigidity. Approximately 32\% of patients in our study were on prostaglandin therapy, and it is possible that this may have affected IOP measurement.

Overall, GAT/DCT differences were positively associated with age (slope $0.05, r^{2}=0.05, \mathrm{p}=0.01$ ) and this relation increased when untreated eyes alone were assessed (slope $\left.0.08, r^{2}=0.14, p=0.003\right)$. In younger eyes, DCT readings were greater than GAT readings, and this difference reversed in older eyes. It has been suggested that age related increase in corneal "stiffness" may induce a further measurement error with GAT, ${ }^{15}$ and studies have shown age related changes that may contribute to this increase. ${ }^{16-18}$ DCT measurements may be less affected as the effect of corneal biomechanics on IOP measurement is reduced with this technique. However, the association found here requires to be validated in further studies powered to detect this effect.

In this study, the intraobserver variability of IOP readings was greater with the DCT compared with the GAT, although opposite findings were reported in a recent paper by Kauffmann et al. ${ }^{7}$ Possible explanations for the difference include differences in study subjects (patients versus hospital staff volunteers), order of testing (randomised order versus GAT followed by DCT), instrument model, and DCT reading quality. The association of reading quality and measurement variability approached significance $(p=0.08)$ in this study, therefore tighter quality control may result in improved repeatability. The better repeatability of GAT in this study may have been because the observers were not masked to their own results. Although the GAT drum was rotated to $10 \mathrm{~mm} \mathrm{Hg}$ between readings, an element of digit preference may have remained. The DCT, on the other hand, provides objective IOP measurements and the operator cannot directly manipulate the readings.

The interobserver variability was relatively low for GAT and DCT measurements, with mean difference (95\% confidence intervals) being 0.4 ( -3.5 to 4.2$) \mathrm{mm} \mathrm{Hg}$ for GAT and 0.2 $(-4.9$ to 5.3$) \mathrm{mm} \mathrm{Hg}$ for DCT. This value is in concordance with most reports of GAT reproducibility. ${ }^{19}$

This study shows that the DCT is less affected by CCT than the GAT. Age accounted for as much intersubject variation in GAT/DCT differences as did CCT, suggesting a significant effect of age related corneal stiffening on IOP measurement with GAT. However, measurement variability was higher with the prototype DCT compared with the GAT. A nonsignificant trend relating reading quality and measurement variation suggests that tonometry technique may be a source of variation.

\section{ACKNOWLEDGEMENTS}

The authors thank Carleton Optical for the loan of the Pascal DCT and Tuan Ho for editing the manuscript. Aachal Kotecha is funded by the Special Trustees of Moorfields Eye Hospital.

\section{Authors' affiliations}

A Kotecha, E T White, J M Shewry, D F Garway-Heath, Glaucoma Research Unit, Moorfields Eye Hospital, London, UK 
Correspondence to: D F Garway-Heath, MD FRCOphth, Glaucoma Research Unit, Moorfields Eye Hospital, 162 City Road, London ECIV 2PD, UK; david.garway-heath@moorfields.nhs.uk

Accepted for publication 3 August 2005

\section{REFERENCES}

1 Goldmann H, Schmidt T. [Applanation tonometry.]. Ophthalmologica 1957:134:221-42.

2 Ehlers N, Bramsen T, Sperling S. Applanation tonometry and central corneal thickness. Acta Ophthalmol (Copenh) 1975;53:34-43.

3 Whitacre MM, Stein RA, Hassanein K. The effect of corneal thickness on applanation tonometry. Am J Ophthalmol 1993;115:592-6.

4 Whitacre MM, Stein R. Sources of error with use of Goldmann-type tonometers. [Review]. Surv Ophthalmol 1993;38:1-30.

5 Gordon MO, Beiser JA, Brandt JD, et al. The Ocular Hypertension Treatment Study: baseline factors that predict the onset of primary open-angle glaucoma. Arch Ophthalmol 2002;120:714-20.

6 Feltgen N, Leifert D, Funk J. Correlation between central corneal thickness, applanation tonometry, and direct intracameral IOP readings. Br J Ophthalmol 2001;85:85-7.

7 Kaufmann C, Bachmann LM, Thiel MA. Comparison of dynamic contour tonometry with goldmann applanation tonometry. Invest Ophthalmol Vis Sci 2004;45:3118-21.

8 Kaufmann C, Bachmann LM, Thiel MA. Intraocular pressure measurements using dynamic contour tonometry after laser in situ keratomileusis. Invest Ophthalmol Vis Sci 2003;44:3790-4.
9 Siganos DS, Papastergiou Gl, Moedas C. Assessment of the Pascal dynamic contour tonometer in monitoring intraocular pressure in unoperated eyes and eyes after LASIK. J Cataract Refract Surg 2004;30:746-51.

10 Bland JM, Altman DG. Statistical methods for assessing agreement between two methods of clinical measurement. Lancet 1986;1:307-10.

11 Bland JM, Altman DG. Statistics notes: measurement error. BMJ 1996;313:744.

12 Bhan A, Browning AC, Shah S, et al. Effect of corneal thickness on intraocular pressure measurements with the pneumotonometer, Goldmann applanation tonometer, and Tono-Pen. Invest Ophthalmol Vis Sci 2002;43:1389-92.

13 Gunvant P, Baskaran M, Vijaya L, et al. Effect of corneal parameters on measurements using the pulsatile ocular blood flow tonograph and Goldmann applanation tonometer. Br J Ophthalmol 2004;88:518-22.

14 Weinreb RN, Toris CB, Gabelt BT, et al. Effects of prostaglandins on the aqueous humor outflow pathways. Surv Ophthalmol 2002;47(Suppl 1):S53-64.

15 Friedenwald JS. Contribution to the theory and practice of tonometry. Am J Ophthalmol 1937:20:985-1024.

16 Daxer A, Misof K, Grabner B, et al. Collagen fibrils in the human corneal stroma: structure and aging. Invest Ophthalmol Vis Sci 1998;39:644-8.

17 Malik NS, Moss SJ, Ahmed N, et al. Ageing of the human corneal stroma: structural and biochemical changes. Biochim Biophys Acta 1992;1138:222-8.

18 Sherrard ES, Novakovic P, Speedwell L. Age-related changes of the corneal endothelium and stroma as seen in vivo by specular microscopy. Eye 1987; 1(Pt 2): 197-203.

19 Tonnu PA, Ho T, Sharma K, et al. A comparison of four methods of tonometry: method agreement and interobserver variability. $\mathrm{Br} J$ Ophthalmol 2005;89:847-50.

\section{1 th European Forum on Quality Improvement in Health Care}

26-28 April 2006, Prague, Czech Republic

For further information please go to: www.quality.bmipg.com

Book early to benefit from a discounted delegate rate 